\title{
Erratum: Optical properties of titanium-doped lithium niobate from time-dependent density-functional theory [Phys. Rev. Materials 1, 034401 (2017)]
}

\author{
Michael Friedrich, W. G. Schmidt, Arno Schindlmayr, and Simone Sanna
}

(Received 13 December 2017; published 11 January 2018)

DOI: 10.1103/PhysRevMaterials.2.019902

In the presentation of our results in Sec. III D, the numerical values of the ordinary $\left(n_{\perp}\right)$ and the extraordinary $\left(n_{\|}\right)$refractive index were inadvertently reversed, affecting the discussion in the main text as well as the figures. Consequently, whenever we refer to our results for the ordinary refractive index or $n_{\perp}$ in the original article, the extraordinary refractive index $n_{\|}$is actually meant, and vice versa. In the revised versions of Figs. 7 and 8 included here, the labels $n_{\perp}$ and $n_{\|}$are now exchanged. Furthermore, we have taken this opportunity to incorporate improved convergence offsets in Fig. 7 that follow the procedure described at the beginning of Sec. III D and slightly raise the numerical values of the refractive indices compared to the original version.

While the error does not affect the conclusions drawn from our results, the dependence of the refractive indices on the titanium concentration in Fig. 8 must be reinterpreted. We find that both the ordinary and extraordinary refractive index are approximately linear functions of the Ti concentration. Minor deviations from the linearity are due to the presence of pairs of Ti atoms situated closely along the crystal $c$ axis in our structural models. Our calculations thus confirm the experimental finding [1,2] that, within the range of the investigated concentrations, the extraordinary refractive index is more strongly affected by Ti indiffusion than the ordinary refractive index. The experimentally observed concave behavior of the ordinary refractive index cannot be reproduced in our simulations due to the finite size of the supercells that can be treated with the required level of accuracy. Indeed, doping concentrations lower than $1.85 \mathrm{~mol} \%$, achieved using the larger $3 \times 3 \times 3$ supercell, are currently not accessible. Moreover, other defect configurations, such as the substitution of $\mathrm{Nb}_{\mathrm{Li}}$ antisites or $\mathrm{Nb}_{\mathrm{Nb}}$ by titanium atoms [3], might in fact be responsible for the experimentally observed deviation from the linear behavior.

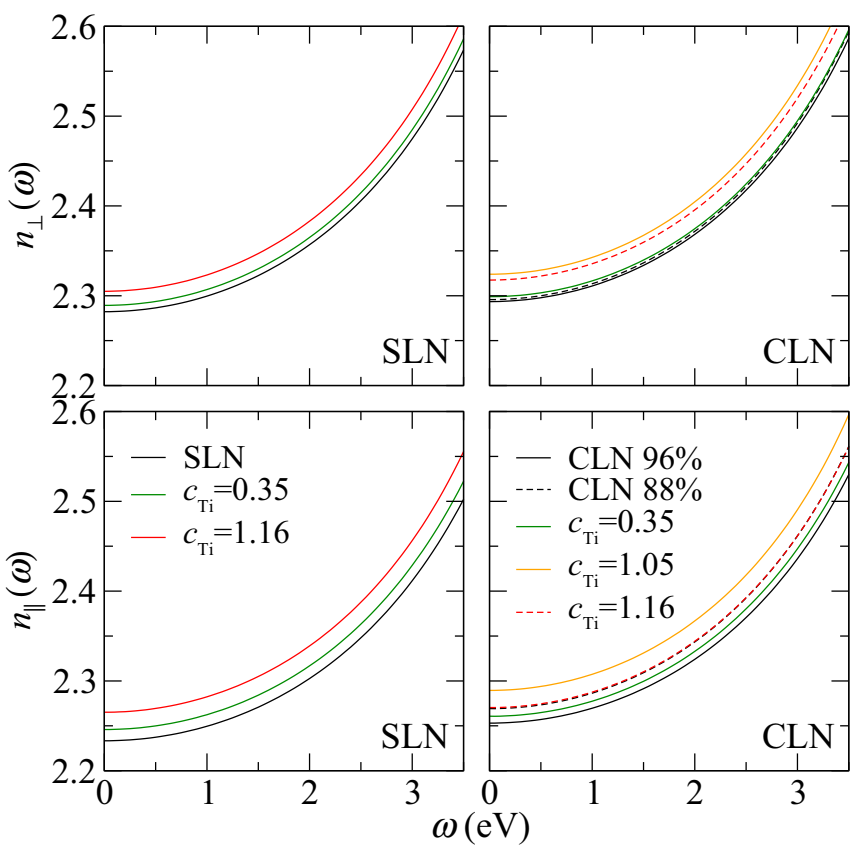

FIG. 7. Refractive indices $n(\omega)$ of stoichiometric and congruent $L N$ with different titanium concentrations. The ordinary $n_{\perp}$ and extraordinary $n_{\|}$components of the refractive indices are shown separately. For information about the impurity concentrations and Li:Nb ratios of the studied systems see the caption of Fig. 5. 


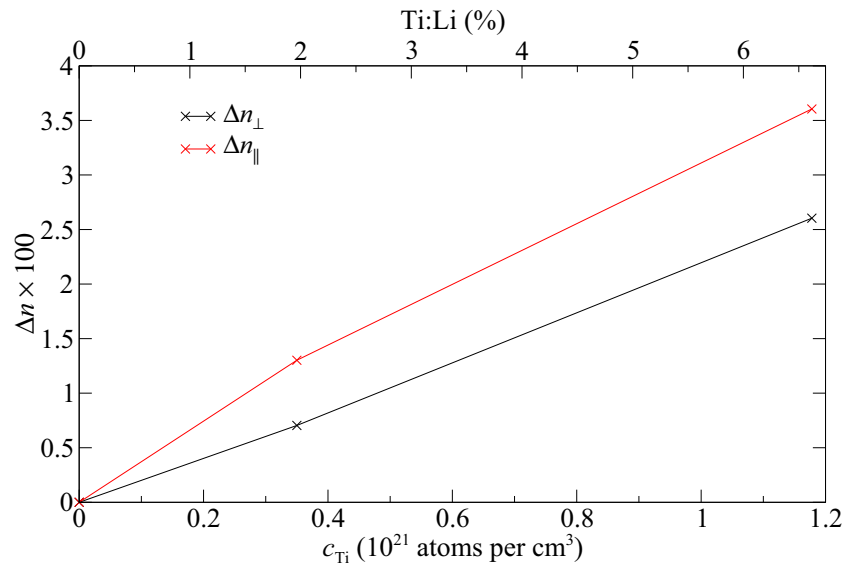

FIG. 8. Variation $\Delta n=n_{\mathrm{Ti}: \mathrm{SLN}}-n_{\mathrm{SLN}}$ of the ordinary $(\perp)$ and the extraordinary $(\|)$ refractive index at a wavelength of $630 \mathrm{~nm}(1.97 \mathrm{eV})$ as a function of the titanium concentration $c_{\mathrm{Ti}}$, given in units of $10^{21}$ atoms per $\mathrm{cm}^{3}$. The calculated data points are joined by lines to guide the eye.

[1] M. Minakata, S. Saito, M. Shibata, and S. Miyazawa, J. Appl. Phys. 49, 4677 (1978).

[2] H. Lüdtke, W. Sohler, and H. Suche, in Digest of Workshop on Integrated Optics, edited by R. T. Kersten and R. Ulrich (Technische Universität Berlin, Berlin, 1980), pp. 122-126.

[3] D. Kollewe, A. Kling, B. C. Grabmaier, T. Bremer, W. Heiland, and W. Zimmermann, Phys. Lett. A 169, 177 (1992). 